\title{
Dependence of target redundancy effects on noise conditions and number of targets
}

\author{
G. ROBERT GRICE and JOHN W. GWYNNE \\ University of New Mexico, Albuquerque, New Mexico
}

\begin{abstract}
In a letter identification task using a four-position array, reaction time (RT) decreased as a negatively accelerated function of the number of targets, both with and without noise letters in nontarget positions. The effect was much greater in the noise condition, in which each redundant target replaced a noise letter. In such situations, the redundant-targets effect should probably be attributed to noise reduction rather than to redundancy. Scaling analysis of the RT distributions indicated that neither noise nor redundancy affects the rate of growth of excitatory strength, but that both affect the time at which growth begins. Excitatory strength grew as a function of the number of redundant targets according to a principle of decreasing gain, with much greater growth in the noise conditions. Further experiments indicated that whether noise is manipulated between or within subjects is important. Unless all stimuli present are reliably targets, redundancy effects are eliminated or reduced in no-noise conditions.
\end{abstract}

In choice reaction time experiments concerned with letter identification, Grice, Canham, and Gwynne (1984) identified conditions under which a redundant-targets advantage did and did not occur when there were two positions in a visual display in which the target letter could appear. Responses were faster in a two-target condition than in a single-target condition in which one position was occupied by a noise letter. However, responses were not faster in a two-target condition than in a single-target condition in which the remaining position was blank. This failure to obtain a redundant-targets effect, relative to a no-noise condition, had been predicted on the basis of the results of a scaling analysis previously conducted by Grice, Canham, and Boroughs (1984). The prediction was confirmed by Grice, Canham, and Gwynne (1984) in two experiments with differing spatial arrangements of the target positions. Since much of the research in this area has been conducted with noise letters present, these results suggest an interpretation somewhat different from the typical one. They suggest that improved performance with two letters is due not specifically to redundancy, but to the reduction in noise when a noise letter is replaced by a target.

However, it must be recognized that the results are of limited generality, because the research was limited to two target positions and either one or two targets. Research in the area has frequently involved more than two targets or more than two positions in which a target may appear. This had been true both in studies of tachistoscopic recognition (Eriksen, 1966; Eriksen \& Lappin, 1965; Kinchla \& Collyer, 1974) and in reaction time (RT) experiments (Estes, 1972; Holmgren, Juola, \& Atkinson, 1974). It seems possible that as subjects' uncertainty increases as to where stimuli may appear, genuine redundancy effects

Portions of this research were supported by Public Health Service Grant MH16400 from the National Institute of Mental Health. Requests for reprints should be addressed to G. Robert Grice, Department of Psychology, University of New Mexico, Albuquerque, NM 87131. may become involved. Thus, the primary purpose of the present research was to investigate a situation with four possible stimulus locations and with from one to four targets present. This situation was examined with and without noise letters present in positions with no target.

A further purpose of this research was to examine the possibility that the effect of noise letters in these situations may depend on whether the presence or absence of noise is manipulated between or within subjects. It seems plausible that, when more than one letter is present, the effect of extra targets may be more useful if all of the letters are always targets, rather than a possible mixture of targets and distractors. In some of our research, using the within-subjects manipulation, we have obtained evidence that a second target may actually be distracting when compared with a single target. Three experiments investigating this problem are presented here.

\section{EXPERIMENTS 1 AND 2}

\section{Method}

Subjects. The subjects were 112 undergraduate women enrolled in introductory psychology courses. They received course credit for participation. There were 28 subjects in each of the two groups of each of the two experiments.

Procedure. The equipment and general procedures were as described by Grice, Canham, and Boroughs (1984). Letter stimuli were presented on the video monitor of a TRS-80 microcomputer. At the viewing distance used, the letters were approximately $.53^{\circ}$ high and $.43^{\circ}$ wide. Four letter positions were located at the corners of an imaginary rectangle. The center-to-center horizontal separation of the positions was $2.0^{\circ}$, and the vertical separation was $1.6^{\circ}$. A plus sign used as the fixation point was located at the center of the rectangle. It went off when the letters appeared and came back on at the end of a trial. There were two target letters, $\mathrm{H}$ and $\mathrm{S}$, and when noise letters were present, they were always $Y$. Subjects were instructed to respond to one target with a left key and to the other with a right key. Half of the subjects were assigned each stimulus-response mapping. Their instructions were to respond as quickly as possible while avoiding errors. A .5-sec tone preceded each trial by $1.0 \mathrm{sec}$. Visual and auditory feedback was given for 
errors. Trials were separated by 4 -sec intervals, and RTs were recorded in milliseconds.

In each condition, there were equal numbers of trials with one, two, three, and four identical targets present. With each number of targets, each possible different spatial arrangement of targets was used equally often. The targets $H$ and $S$ were used equally often, and all of the events occurred in an irregular order. Experiments began with 50 practice trials followed by a brief rest. There were then 250 trials without interruption. Data were based on the final 240 trials, 60 with each number of targets.

There were two groups of subjects in each of the two experiments. For one group, the noise letter appeared in each position with no target. For the other group, these positions were blank. The two experiments differed only in the duration of the stimulus display. In Experiment 1, the display was terminated by the response. In Experiment 2, the display was on for $150 \mathrm{msec}$. The purpose of this difference was to evaluate the possible role of eye movements. A 150 -msec duration is too brief to permit a response based on a fixation following a saccade.

\section{Results}

Mean reaction time. The only data of interest are correct responses, inasmuch as error rates were low. The 16 mean error rates of the two experiments varied from .007 to .030 , with a mean of .016 . There was a slight tendency for error rate to decrease with the number of targets. For one, two, three, and four targets, the error rates were $.023, .017, .013$, and .011 , respectively. No other systematic tendencies were present for errors.

Mean RTs for correct responses as a function of number of targets for the noise and no-noise conditions of the two experiments are presented in Figure 1. In all conditions, RT decreased as a negatively accelerated function of the number of targets. The primary effect of number of targets was significant $[F(3,324)=148.35, p<.001]$. In other words, improvement with the number of targets occurred according to a principle of decreasing gain. This was true both with and without noise letters present, but RT was slower in the noise condition. However, as the number of targets was increased, the amount of improvement was greater with noise present. Both of these effects were statistically significant [for the noise effect, $F(1,108)=6.53, p<.025$; for the noise $\times$ number of targets interaction, $F(3,324)=29.45, p<.001]$. Although RTs in Experiment 1, with the responseterminated stimuli, were a little slower than those in Experiment 2 , both with and without noise, the effect of stimulus duration was not significant $[F(1,108)=1.20$, n.s.]. Furthermore, none of the interactions involving experiments approached significance. This means that the opportunity for eye movements during the trial was not a matter of significance in this situation.

In view of the finding of Grice, Canham, and Gwynne (1984) that a redundant-targets effect was not obtained in the two-position situation without noise, a major point of interest here is whether the smaller effect observed in the no-noise conditions was statistically significant. The answer is that, in the no-noise conditions alone, the number-of-targets effect was significant $[F(3,162)=$ $30.91, p<.001]$. The difference between the two stimulus durations was not significant $[F(1,54)=1.07$, n.s.].

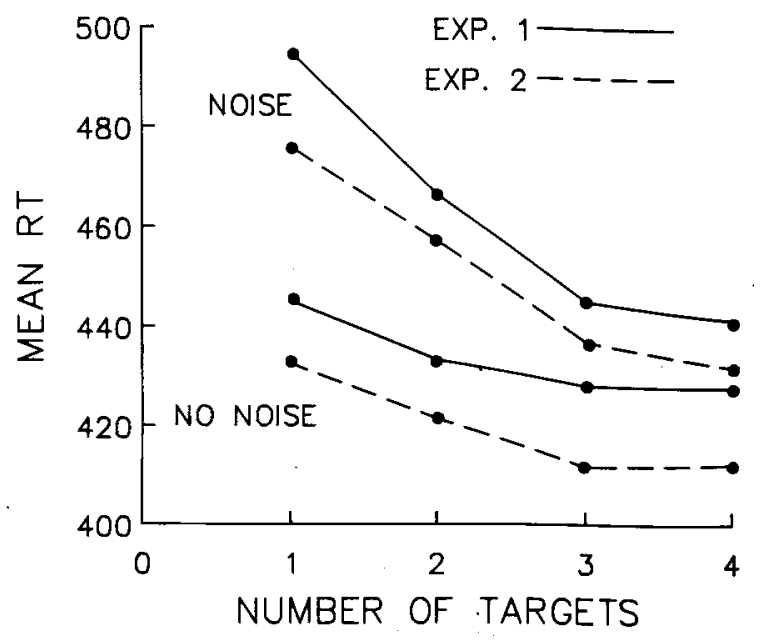

Figure 1. Mean reaction time (RT) for each number of targets and each noise condition in Experiments 1 and 2.

Also, the interaction of experiments $\times$ number of targets was not significant $[F(3,162)<1]$. The results differ from those of Grice, Canham, and Gwynne (1984) with the two-position experiments. It is worth remembering, however, that in the earlier research the presence of noise was manipulated within subjects, whereas in the present experiments it was manipulated between subjects.

One feature of possible interest is that the four-target RTs for the noise conditions were slower than those for the no-noise conditions in both experiments. Of course, the displays were actually the same in both conditions. Although such a result may not be surprising, in view of possible criterion effects, the difference was not significant $[F(1,108)=1.95$, n.s. $]$.

Relevant to the interpretation of such experiments is the role of individual target positions. It is possible that effects of multiple presentation may be due to the increased probability of a target's appearing in a favored position, rather than to redundancy per se. The experiments reported here are not ideally suited to such an analysis, inasmuch as numbers of trials were equated for numbers of targets and not for target positions. However, we did conduct limited analyses of this kind, restricted to the nonoise conditions.

In Experiments 1 and 2, mean RTs to the four singletarget positions did not differ significantly $[F(3,162)=$ 2.12 , n.s.]. However, it has been suggested that the favored position may differ between subjects, and that the appropriate comparison for redundant-target conditions would be with the mean of the fastest single-target position for each subject. To this end, we identified the fastest single-target position for each of the 56 subjects. The first application of these data was to the two-target data. For each subject, the data were separated into two-target combinations that did and did not include the subject's fastest single position. The means of these two groups of trials differed by only $1 \mathrm{msec}$ and clearly did not support the interpretation that RT depended on the presence of a favored position. 
The second application of a fastest-position analysis was a comparison with the four-target condition. The mean RT for the fastest one-target position was actually faster than the mean RT for the four-target condition. However, this must be viewed with caution. Biederman and Checkosky (1970), who first used this logic, pointed out, in effect, that if a single condition is selected for comparison on the basis of speed, its value may depend largely on sampling error. Thus, the proper comparison is with the fastest of an equal number of redundant conditions. Therefore, we randomly divided the four-target trials into four blocks of 15 trials, equal to the number of trials for each single-target position, and identified the fastest block for each subject. The mean RT for the fastest one-target position was $413 \mathrm{msec}$, and that for the fastest four-target block was $395 \mathrm{msec}$. This difference is significant $[F(1,54)=48.75, p<.001]$, and confirms the conclusion based on the data as a whole.

Another position-related factor of possible relevance is that of stimulus-response compatibility. For example, Grice, Canham, and Boroughs (1984) and Grice, Canham, and Gwynne (1984), in two-position experiments, found advantages ranging from 23 to $45 \mathrm{msec}$ when a single target was on the same side as the response key, as opposed to the opposite side. This was evaluated here for the single- and two-target conditions. When the single target was on the same side of the display as the response, mean RT was $435 \mathrm{msec}$; when it was on the opposite side, mean RT was $442 \mathrm{msec}$. This 7 -msec effect was significant $[F(1,54)=4.80, p<.05]$, but was substantially less than that in the two-position experiments. In two-target displays, when both were in the compatible position, mean RT was $430 \mathrm{msec}$; when both were in the incompatible position, mean RT was $435 \mathrm{msec}$. The difference was not significant $[F(1,54)<1]$. It is our conclusion that compatibility effects were essentially negligible in this situation.

Scaling analysis. One approach to examining the dynamics of the redundant-signals effect is the application of the scaling analyses of variable criterion theory to the RT distributions (Grice, Canham, \& Boroughs, 1984). Following the procedures used previously, we obtained functions for the growth of excitatory strength from the cumulative distributions for each stimulus and noise condition in each of the two experiments. As in the previous research, the function, in scale units, for the singlestimulus conditions could be described by exponential growth functions of the following form:

$$
E=a-m e^{-k t} .
$$

In this function, $E$ refers to excitatory strength, and $t$ refers to time in milliseconds from stimulus onset. The asymptote is $a$, and $m$ is the total amount of growth from intercept to asymptote. It is a property of this function that, at any time, the function grows as a constant proportion of the remaining distance to asymptote per unit of time. The parameter determining this proportional growth rate is the exponential constant $k$. The function may also be written in the form

$$
E=m\left(1-e^{-k t}\right)+c,
$$

where $c$ is the intercept. The growth functions used in this analysis were based on the cumulative distributions for each number of targets with all target positions combined. The above function for excitatory strength was first fitted to the one-target, no-noise condition of Experiment 1 . The parameters for Equation 1 were $a=2.239$, $m=21.868$, and $k=.00536$. In the form of Equation 2, the value of $c$ was -19.629. Data points were at the 19 mean quantiles at .05 intervals. The proportion of variance accounted for was greater than .999 .

Grice, Canham, and Boroughs (1984) and Grice, Canham, and Gwynne (1984) found that the growth functions for two targets were linearly related to the average of the two single-target functions. Thus, a logical beginning for the present analysis was to examine the relation of the single-target function to all of the remaining functions. This was done by computing the fitted single-target function at the 19 quantiles of all of the other functions. The obtained scale values at these times, for each of the remaining 15 conditions, were then related to these computed scale values. The resulting relationships were all linear. This outcome has a clear interpretation. If two functions such as Equation 1 or Equation 2 are related to each other at corresponding times, the result will be linear if the proportional growth rate, as indicated by the parameter $k$, is the same for the two functions. The outcome here means that the proportional growth rate is invariant with number of targets, presence or absence of noise, and stimulus duration. The invariance of proportional growth rate does not mean that this is also true of absolute growth rate, which also depends on the amount of total growth as indicated by the parameter $m$.

The results of this analysis are summarized in Table 1, which shows the slopes and intercepts, as well as the values of $r^{2}$, for the linear functions relating the obtained scale values of the 15 conditions to the calculated values of the single-target fitted function. The values of $r^{2}$ indicate the degree to which the data support the finding of invariance of the parameter $k$. Although there is no appropriate error term for evaluating possible significance of departure from linearity, it may be noted that the mean proportion of variance available for nonlinearity is .0014. The intercepts of the linear relations indicate the general level of the strength functions at the same times as that of the single target, no-noise condition. In terms of the distributions, they reflect the central tendency. The correlation of the intercepts with the mean RTs of Figure 1 is .996. Like the means, the intercepts are significantly affected both by the number of targets and by noise [for number of targets, $F(3,6)=296.76, p<.001$; for number of targets $\times$ noise, $F(3,6)=56.78, p<.001]$. In a comparison of the three target conditions in which noise was either present or absent, the effect of noise is significant $[t(5)=7.851, p<.001]$.

The slope values indicate the absolute rates of growth in strength, relative to the single-target condition. An inspection of the table indicates a clear tendency for the 
Table 1

Slopes, Intercepts, and Values of $\boldsymbol{r}^{2}$ for Linear Functions Relating the Growth Function for Each Condition to the Calculated Function for the Single Target, No-Noise Condition of Experiment 1

\begin{tabular}{|c|c|c|c|c|c|c|c|}
\hline \multirow[b]{2}{*}{ Experiment } & \multirow{2}{*}{$\begin{array}{l}\text { Number of } \\
\text { Targets }\end{array}$} & \multicolumn{3}{|c|}{ No Noise } & \multicolumn{3}{|c|}{ With Noise } \\
\hline & & Slope & Intercept & $r^{2}$ & Slope & Intercept & $r^{2}$ \\
\hline 1 & 1 & 1.000 & .000 & .999 & 1.081 & -.505 & .999 \\
\hline 1 & 2 & .995 & .117 & .999 & 1.029 & -.222 & .999 \\
\hline 1 & 3 & .948 & .195 & .999 & .994 & -.022 & .999 \\
\hline 1 & 4 & .964 & .182 & .998 & .947 & .044 & .999 \\
\hline 2 & 1 & 1.042 & .136 & .999 & 1.123 & -.386 & .998 \\
\hline 2 & 2 & 1.048 & .250 & .999 & 1.079 & -.167 & .995 \\
\hline 2 & 3 & .978 & .376 & .999 & .985 & .075 & .998 \\
\hline 2 & 4 & .954 & .357 & .999 & 1.006 & .167 & .999 \\
\hline
\end{tabular}

slopes to decrease as the number of targets increases $[F(3,6)=20.08, p<.005]$. This appears to lead to the surprising conclusion that excitatory strength grows more slowly with increasing target redundancy. Further inspection of Table 1 indicates that the same apparently paradoxical relation applies to the effect of noise. With number of targets constant, the slopes and growth rates are greater with noise than with no noise $[t(5)=3.881$, $p<.02]$. What this picture means is that the effects of noise and target redundancy are both short-latency phenomena that begin to have their effects before responses occur. Thus, conditions with more targets and without noise produce responding and rapid growth of strength before less favorable conditions. The proportional growth rate is unaffected by these variables, and since, at any time, the more favorable condition is closer to asymptote, the absolute growth rate is less. Target redundancy and the presence or absence of noise affect not the rate of growth of excitatory strength, but the time at which growth occurs. This model is presented graphically for Experiment 1 in Figure 2. The growth functions are computed from the function fitted to the single-target condition by means of the linear transformations of Table 1 . The normal functions indicate the assumed normally distributed criterion of variable criterion theory. The cumulative proportion of response at any time is the area of the normal function below the ordinate of a growth function at that time.

In terms of the distributions on which the scaling solution was based, the implication of this analysis is that the effects of both number of targets and noise are almost entirely on central tendency. Effects on the shape and variability of the distribution are negligible.

The scaling procedures also allowed us to examine the manner in which excitatory strength is determined by the number of redundant targets. In a simple detection task, Kinchla and Collyer (1974) hypothesized that strength, in a normal metric, would be a linear function of the number of targets. Purely on the basis of logical considerations, however, we would expect the function here to be one of decreasing gain. Because strength increased throughout the RT distribution, the function we sought was not a single one, but a series of such functions at various times following stimulus onset. This analysis was ap- plied to Experiment 1, although the results are about the same for Experiment 2.

We began by fitting a function to the values of $E$ for the noise condition at $500 \mathrm{msec}$ as a function of the number of redundant targets. The function turned out to be a negatively accelerated exponential function similar to Equations 1 and 2. However, in this instance we used a form somewhat more suitable for use with a discrete variable, as follows:

$$
E=m\left[1-(1-\theta)^{N}\right]+c .
$$

In this equation, $E$ is excitatory strength, $N$ is the number of redundant targets, $m$ and $c$ have the same meaning as in Equation 2, and $\theta$ is the actual proportional growth constant for each additional target. For the fitted function, $\theta=.472, m=.545$, and $c=.290$. We next examined the relation of this function to the obtained functions at $340,400,600$, and $700 \mathrm{msec}$, for both the noise and no-noise conditions. All of these relations were linear

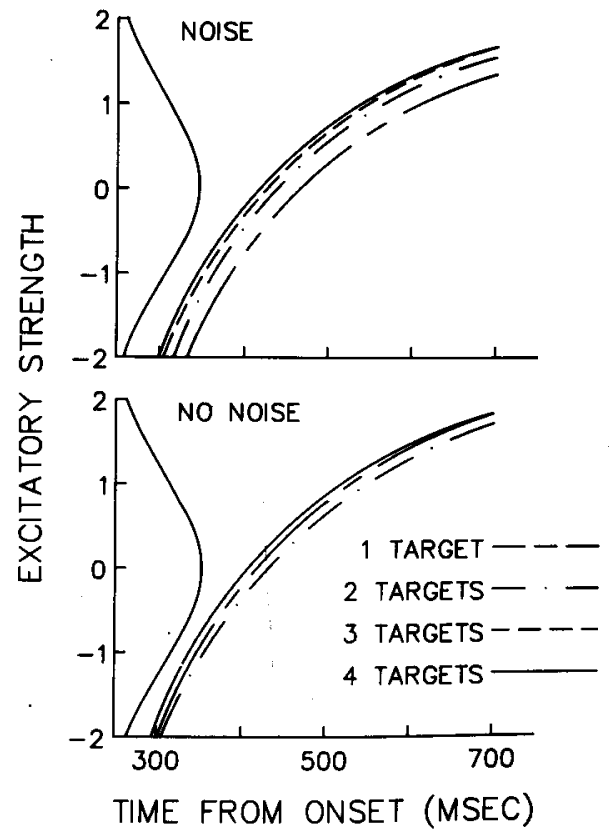

Figure 2. Calculated functions for the growth of excitatory strength for Experiment 1. 
for corresponding numbers of targets. From these linear parameters, the fitted function was transformed to all of the other conditions. The results are plotted, together with the data points, in Figure 3.

These results clearly show that the redundant-targets function not only follows a principle of decreasing gain, but does so in a remarkably consistent fashion. The proportional rate of growth to asymptote is constant throughout the entire RT distribution, both with and without noise letters in the no-target positions. Aside from the absolute level of the functions, they differ in the amount of growth as indicated by the parameter $m$. The greatest effects of redundancy are at short latencies, and with noise present. Of course, with noise, each redundant target also meant the elimination of a noise letter. To emphasize the effect of both time and the presence of noise, we have plotted, in Figure 4, the parameter $m$ as a function of time for the noise and no-noise conditions separately. Since $\theta$ is constant, it is entirely $m$ that determines the slope of the function for number of redundant targets.

\section{EXPERIMENTS 3 AND 4}

The presence and absence of noise were investigated with separate groups of subjects in Experiments 1 and 2. It was not practical to study both number of targets and noise as within-subjects effects. However, in Grice, Canham, and Gwynne's (1984) previous experiments with two targets, the subjects experienced equal numbers of noise and no-noise trials. Thus, the present Experiments 1 and 2 and the previous experiments were not strictly comparable except for the numbers of targets and target positions. For this reason, we conducted two additional experiments, using four target positions. In both of these experiments, we studied noise within subjects. In one we compared one and two targets, and in the other we compared one and three targets.

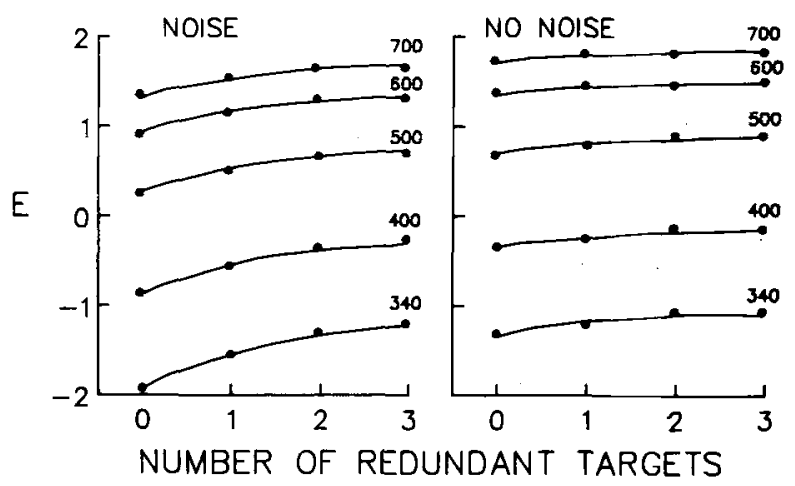

Figure 3. Calculated functions and data points indicating dependence of excitatory strength (E) on the number of redundant targets. Times of the functions are at $340,400,500,600$, and 700 msec following stimulus onset.

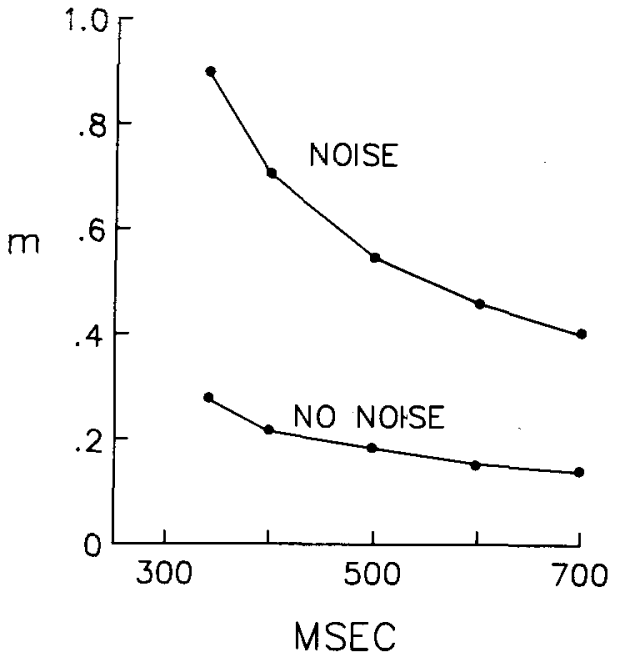

Figure 4. Total growth of excitatory strength from intercept to asymptote $(\mathrm{m})$ of the functions for number of redundant targets. Points are at the times indicated in Figure 3 for the noise and nonoise conditions.

\section{Method}

There were 28 subjects, drawn from the same population as the subjects in Experiments 1 and 2, in each experiment. The general procedures and the visual display were the same as in Experiment 1, with response-terminated stimuli. In Experiment 3, half of the trials were with single targets and half were with two targets. For each number of targets, half of the trials were with noise letters in nontarget positions and half were without noise. As in Experiments 1 and 2, all of the letter positions were used equally often. Experiment 4 differed only in that the two-target trials were replaced with three-target trials.

\section{Results}

Mean RTs for correct responses for Experiments 3 and 4 are presented in the lower portion of Table 2. The comparable means from Experiment 1 for one, two, and three targets are presented in the upper portion of the table. In Experiment 3, for the noise condition, RT was significantly faster for two targets than for one target $[t(27)=$ $7.15, p<.001]$. Also, for Experiment 4, for the noise condition, RT was significantly faster for three targets than for one target $[t(27)=9.13, p<.001]$. These differences are both about the same size as the corresponding differences of Experiment 1.

As hypothesized, however, redundancy was less effective in the no-noise condition if the subjects experienced both noise and no-noise trials. The difference between one and two targets in the no-noise condition of Experiment 3 was only $3 \mathrm{msec}$ and was not significant $[t(27)<1]$. The difference between one and three targets in Experiment 4 was just $8 \mathrm{msec}$, and did not reach significance at the .05 level $[t(27)=1.85, .10>p>.05]$. These differences are both smaller than the corresponding differences in the no-noise condition of Experiment 1. When the one- and 
Table 2

Mean Correct Reaction Time (in Milliseconds) as a Function of Number of Targets, With and Without Noise Letters (Presence and Absence of Noise Manipulated Both Between and Within Subjects)

\begin{tabular}{|c|c|c|c|c|c|c|}
\hline & \multicolumn{3}{|c|}{ No Noise } & \multicolumn{3}{|c|}{ With Noise } \\
\hline & $\begin{array}{c}\text { One } \\
\text { Target }\end{array}$ & $\begin{array}{c}\text { Two } \\
\text { Targets }\end{array}$ & $\begin{array}{l}\text { Three } \\
\text { Targets }\end{array}$ & $\begin{array}{c}\text { One } \\
\text { Target }\end{array}$ & $\begin{array}{c}\text { Two } \\
\text { Targets }\end{array}$ & $\begin{array}{c}\text { Three } \\
\text { Targets }\end{array}$ \\
\hline \multicolumn{7}{|c|}{ Noise Between Subjects* } \\
\hline $\begin{array}{l}\text { Mean } \\
S D\end{array}$ & $\begin{array}{r}445 \\
56\end{array}$ & $\begin{array}{r}433 \\
51\end{array}$ & $\begin{array}{r}428 \\
60\end{array}$ & $\begin{array}{r}496 \\
89\end{array}$ & $\begin{array}{r}466 \\
83\end{array}$ & $\begin{array}{r}445 \\
83\end{array}$ \\
\hline \multicolumn{7}{|c|}{ Noise Within Subjects $\dagger$} \\
\hline $\begin{array}{l}\text { Mean } \\
S D\end{array}$ & $\begin{array}{r}437 \\
63\end{array}$ & $\begin{array}{r}434 \\
62\end{array}$ & & $\begin{array}{r}489 \\
68\end{array}$ & $\begin{array}{r}460 \\
62\end{array}$ & \\
\hline Mean & 451 & & 443 & 519 & & 468 \\
\hline$S D$ & 67 & & 66 & 72 & & 68 \\
\hline
\end{tabular}

*Experiment 1. †Experiments 3 and 4.

two-target differences are compared across experiments, the experiments $\times$ number of targets interaction is significant $[F(1,54)=3.75, p<.05$, one-tailed test $]$. However, when the differences between one and three targets are compared, the interaction, although in the same direction, does not reach significance $[F(1,54)=2.74$, n.s.].

In the case of Experiment 4, it was possible to conduct an appropriate comparison of the fastest one-target and three-target conditions. There were four single-target positions and four three-target arrangements. For each subject, the fastest of these conditions was identified for the no-noise condition. For comparison, the same data were obtained from the corresponding conditions of Experiment 1. In Experiment 1, the mean RT for the fastest onetarget position was $418 \mathrm{msec}$, and the mean RT for the fastest three-target arrangement was $402 \mathrm{msec}$. The difference was significant $[t(27)=4.016, p<.001]$. The mean RTs for Experiment 4 were $428 \mathrm{msec}$ for one target and $423 \mathrm{msec}$ for three targets. This difference was not significant $[t(27)=1.201$, n.s. $]$. In a comparison of the two experiments, the experiments $\times$ number of targets interaction reached significance $[F(1,54)=2.91$, $p<.05$, one-tailed test]. These results support the conclusion that the presence of noise on some trials impaired the effectiveness of redundant stimuli.

Error rates in Experiments 3 and 4 were low, with a mean of .016 .

\section{EXPERIMENT 5}

Following the findings of Grice, Canham, and Gwynne (1984) that redundant-targets effects were not obtained without noise in displays limited to two target positions, we hypothesized that these effects might be more probable with greater position uncertainty. In Experiments 1 and 2, we did find these effects, both with and without noise, in a four-position display. However, these results were obtained with between-subjects manipulation of noise, whereas the original two-position experiments had studied noise within subjects. In Experiments 3 and 4, we failed to find significant redundant-targets effects with the four-position display when subjects experienced both noise and no-noise trials. Together, these findings suggested the possibility that we might obtain the redundanttargets advantage in a two-position display with subjects who never experienced noise letters in the no-target position. This possibility was somewhat enhanced by recent positive findings of van der Heijden, Schreuder, Maris, and Neerincx (1984), who used a somewhat different task. In Experiment 5 we examined this possibility in a twoposition task, with separate groups of subjects with and without noise letters in the one-target displays.

\section{Method}

Experiment 5 was essentially a separate-groups replication of Grice, Canham, and Gwynne's (1984) Experiment 2. The stimuli were the same as those used in that experiment and in Experiments 1-4 of the present study. Stimulus duration was $150 \mathrm{msec}$. There were two target positions vertically separated by $3^{\circ}$, with the fixation point halfway between. Each position was used equally often on one-target trials. There were equal numbers of trials with the target in the upper position, the lower position, and both positions. The total number of trials and the general procedures were the same as in the other experiments reported here. For one group of subjects, the noise letter was present in the position with no target, and for the other group of subjects, this position was blank. The subjects were from the same population as those of Experiments 1-4 of the present study; this was the same population used by Grice, Canham, and Gwynne. There were 28 in each group.

\section{Results}

Mean RTs for Experiment 5 are presented in the lower portion of Table 3 . The upper portion of the table presents the corresponding means from Experiment 2 of Grice, Canham, and Gwynn (1984). A surprising feature of the data of Experiment 5 is that the noise condition was a bit faster than the no-noise condition. However, this betweensubjects effect was not significant, and all it means is that there was no significant primary effect of noise $[F(1,54)<1]$. The effect of target condition was significant $[F(2,108)=31.58, p<.001]$. This is primarily due to faster response with two targets. The target location $X$ noise interaction was also significant $[F(2,108)=7.88$, $p<.001]$. This reflects the fact that the redundant-targets

Table 3

Mean Correct Reaction Time (in Milliseconds) for Upper, Lower, and Both Target Positions

\begin{tabular}{|c|c|c|c|c|c|c|}
\hline & \multicolumn{3}{|c|}{ No Noise } & \multicolumn{3}{|c|}{ With Noise } \\
\hline & Upper & Lower & $\begin{array}{c}\text { Both } \\
\text { Positions }\end{array}$ & Upper & Lower & $\begin{array}{c}\text { Both } \\
\text { Positions }\end{array}$ \\
\hline \multicolumn{7}{|c|}{ Noise Within Subjects* } \\
\hline $\begin{array}{l}\text { Mean } \\
S D\end{array}$ & $\begin{array}{r}433 \\
68\end{array}$ & $\begin{array}{r}437 \\
69\end{array}$ & $\begin{array}{r}437 \\
69\end{array}$ & $\begin{array}{r}468 \\
81\end{array}$ & $\begin{array}{r}471 \\
82\end{array}$ & $\begin{array}{r}437 \\
69\end{array}$ \\
\hline \multicolumn{7}{|c|}{ Noise Between Subjects† } \\
\hline $\begin{array}{l}\text { Mean } \\
S D\end{array}$ & $\begin{array}{r}440 \\
67\end{array}$ & $\begin{array}{r}446 \\
70\end{array}$ & $\begin{array}{r}433 \\
66\end{array}$ & $\begin{array}{r}424 \\
46 \\
\end{array}$ & $\begin{array}{r}444 \\
58 \\
\end{array}$ & $\begin{array}{r}405 \\
40 \\
\end{array}$ \\
\hline
\end{tabular}

*From Grice, Canham, and Gwynne (1984, Experiment 2). $\nmid$ Experiment 5. 
effect was larger with noise present. This result has been obtained in all experiments. The mean error rate for the experiment was .014 .

The major feature of interest in this experiment is that a redundant-targets effect may have occurred for subjects who did not experience noise during the experiment. In the no-noise group, the 7-msec superiority of the twotarget condition over the single-target, upper-position condition was hardly enough to arouse interest. However, this small difference did approach statistical significance $[t(27)=1.89, .10>p>.05]$. It may be noted that the corresponding difference in the within-subjects experiment was in the opposite direction. This experiments $\times$ number of targets interaction reached significance $[F(1,54)=$ $5.61, p<.025]$.

We also compared the two-target position with the faster single-target position for each subject separately. The mean single-target RT for the no-noise group was $434 \mathrm{msec}$, only $1 \mathrm{msec}$ slower than the two-target RT, so the effect was definitely not obtained according to this criterion. When Grice, Canham, and Gwynne (1984) made this comparison in their noise-within-groups experiment, they found that RT to two targets was $10 \mathrm{msec}$ slower than RT to the faster single position without noise, and this difference was significant. When these differences are compared, the resulting experiments $\times$ number of targets interaction is again significant $[F(1,54)=6.26$, $p<.025]$. We take this to mean that a second target will produce a small amount of interference if the second letter is equally likely to be a distractor or a target. If two letters are reliably both targets, no interference is produced; however, the redundant-targets advantage is either absent or minimal in this situation.

These results are in apparent conflict with the results of van der Heijden et al. (1984), who found a statistically significant redundancy effect when the mean for two targets was compared with the mean of the faster subject positions for single targets. Their experiment was also a two-position experiment with no noise present. However, their experiment differed from ours in that they used the disjunctive or go/no-go procedure, rather than choice reaction time. It is probably well to consider that the differences between the two procedures may be more apparent than real. Van der Heijden et al.'s obtained effect was small, only $11 \mathrm{msec}$, and differs from the result of the present experiment by only $10 \mathrm{msec}$. It may be noted that the caution of Biederman and Checkosky (1970) about comparing the faster of two single positions with a single redundant condition was not observed either in the above analysis or by van der Heijden et al. (1984). In other words, both may have slightly underestimated a redundancy effect. As an approximation of Biederman and Checkosky's suggestion, we separated, at random, the 80 two-target trials into two groups of $\mathbf{4 0}$ trials, and obtained the faster RT in each group for each subject. The mean of these faster trials was $425 \mathrm{msec}$, compared with a mean of $434 \mathrm{msec}$ for the faster single-target position. This unimpressive 9-msec difference reached significance $[t(27)=2.508, p<.02]$. We do not regard this test as fully adequate, since the two-target data were based on fewer trials and thus were less reliable than the singletarget data. However, it does suggest that a relatively trivial redundancy effect was present in the data.

\section{DISCUSSION}

The data of Experiments 1 and 2 confirmed the hypothesis that a redundant-targets advantage would be obtained for both noise and no-noise conditions when the number of possible positions was increased beyond two. This outcome, however, requires qualification. Experiments 3 and 4 indicated that the effect for the no-noise condition was eliminated or substantially reduced for subjects who experienced both redundant targets and noise letters on trials when more than one letter was present. Thus, the obtaining of this effect depends on the noise context in which the redundant stimulus trials occur. Unless all of the stimuli present are always targets, the effect is eliminated or reduced.

The effects of redundancy and noise are intimately related. Experiments 1 and 2 showed that the effect of number of targets is substantially greater in the noise condition. In the noise condition, it is significant that a noise element is eliminated when a redundant target is added. In a comparison of the noise and no-noise conditions, the data indicate that the elimination of a noise letter is quantitatively more effective than the addition of a redundant target. Not all of the research on redundancy has utilized noise stimuli. However, in the literature in general, it is very likely true that effects attributed to redundancy frequently should have been attributed to noise reduction, rather than to redundancy per se.

Two major conclusions followed from the scaling analysis of the RT distributions of Experiments 1 and 2. The first was that neither noise nor redundancy affect the rate of growth of excitatory strength as a function of time. Rather, they affect the time at which growth occurs. The effect of noise is to delay the beginning of growth of excitatory strength, but once responding begins, the rate of growth is not affected. Similarly, the effect of redundancy, although smaller, is an earlier beginning of excitatory strength, but not a faster rate of growth. In less theoretical terms, it is the central tendency and not other properties of the RT distribution that is affected by noise and redundancy.

The second result of the scaling analysis was the set of functions describing the relation between excitatory strength and the number of redundant targets. At any time following stimulus onset, each additional target contributes an increment of strength that is a constant proportion of the distance from asymptote of the number-oftargets function at that point. This proportional increment remains invariant at all points of the distribution, and whether or not the target replaces a noise letter. Although the proportional increment is constant, the absolute increments vary with time and the presence or absence of noise. 
The absolute increment depends on the total growth of the function, which is greatest early in the interval and substantially greater when the early portion of the function is depressed by noise. This is the relation displayed in Figure 4, which shows the total growth of the numberof-redundant-targets functions from intercept to asymptote. In terms of strength, these functions clearly show the minor effect of number of targets in the no-noise condition, compared with the noise condition, in which each redundant target also replaces a noise element.

Experiments 3 and 4 and Experiment 5 are supplementary and consistent in showing the difference between within-subjects and between-subjects manipulation of noise in experiments of this kind. In the four-position situation with no noise, within-subjects manipulation of noise eliminated or reduced the redundant-targets advantage. In the two-position condition of Experiment 5, we did not obtain a convincing redundant-targets advantage with between-subjects noise. However, we did eliminate the interference effect of a redundant target, an effect that had been obtained in two within-subjects experiments by Grice, Canham, and Gwynne (1984). The experiments are consistent in showing that the effect of redundant targets is impaired if, on other multiple-target trials, noise stimuli are present with a target. The results of Experiment 5, in comparison with Experiments 1 and 2, tend to confirm our original hypothesis that the possibility of a redundant-targets effect would increase as position uncertainty increased. However, this result seems to be limited to conditions in which subjects do not also experience noise along with targets.

The so-called redundant-targets advantage has been reliably obtained in two-position experiments when a single target paired with noise is compared with two targets.
Perhaps such experiments are misnamed, and the effect should be viewed merely as an instance of noise reduction. For example, in the experiments of Grice, Canham, and Gwynne (1984), RTs to single targets accompanied by a noise letter were significantly slower than RTs to single targets alone.

\section{REFERENCES}

Biederman, I., \&heckosky, S. F. (1970). Processing redundant information. Journal of Experimental Psychology, 83, 486-490.

ERIKSEN, C. W. (1966). Independence of successive inputs and uncorrelated error in visual form perception. Journal of Experimental Psychology, 72, 26-35.

ERIKSEN, C. W., \& LAPPIN, J. S. (1965). Internal perceptual system noise and redundancy in simultaneous inputs in form identification. Psychonomic Science, 2, 351-352.

ESTES, W. K. (1972). Interactions of signal and background variables in visual processing. Perception \& Psychophysics, 12, 278-286.

Grice, G. R., Canham, L., \& Boroughs, J. W. (1984). Combination rule for redundant information in reaction time tasks with divided attention. Perception \& Psychophysics, 35, 451-463.

Grice, G. R., Canham, L., \& GWYNNe, J. W. (1984). Absence of a redundant-signals effect in a reaction time task with divided attention. Perception \& Psychophysics, 36, 565-570.

Holmgren, J. E., Juola, J. F., \& AtKinson, R. C. (1974). Response latency in visual search with redundancy in the visual display. Perception \& Psychophysics, 16, 123-128.

KinCHLA, R. A., \& Collyer, C. E. (1974). Detecting a target letter in briefly presented arrays: A confidence rating analysis in terms of a weighted additive effects model. Perception \& Psychophysics, 16, 117-122.

van der Heideen, A. H., Schreuder, R., Maris, L., \& Neerincx, M. (1984). Some evidence of correlated separate activation in a simple letter detection task. Perception \& Psychophysics, 36, 577-585.

(Manuscript received September 8, 1986; revision accepted for publication February 9, 1987.) 